\title{
Studying the impact of merged and divided storage policies on the profitability of a remanufacturing system with deteriorating revenues
}

\author{
Hamed Samarghandi \\ Department of Finance and Management Science \\ Edwards School of Business, University of Saskatchewan \\ Saskatoon, SK, Canada
}




\section{Studying the impact of merged and divided storage policies on the profitability of a remanufacturing system with deteriorating revenues}

\section{Abstract}

Merging capacity for a remanufacturing system is studied in this paper. In the system under study, there are two streams for returns and each stream has its dedicated processing line. However, the storage space is merged between the streams. Two strategies are investigated and compared in this paper. The first strategy is to divide the storage space between the two streams in the way that each type of return has its predetermined space in the storage area (divided capacity). In the second strategy, storage space is not split between the two streams and each unit of return, independent of its type, is admitted if there is vacant space (merged capacity). In both strategies, the value of remanufactured products decreases over time by a known factor called the decay rate. Mathematical models to maximize the total profit in each strategy is presented and also verified by a simulation model. From a practical point of view, selecting the correct strategy is an important decision for the remanufacturers because choosing the wrong policy leads to lost profits. Numerical experiments reveal that neither of the scenarios is always preferred to the other one and the 
choice of the optimal strategy depends on the parameters' values and product types. For instance, increasing the remanufacturing cost of the superior product, or increasing the sale price of the inferior product make the merged storage strategy more desirable. On the contrary, increasing the remanufacturing cost of the inferior product, or increasing the sale price of the superior product make the divided storage policy more appealing.

Keywords: Remanufacturing system; Capacity merging; Capacity allocation; Mathematical model; Queuing system

\section{Introduction}

Remanufacturing, as the core business in the reverse supply chain, is the process of recovering the value of the used products. Profitability, legislation and social responsibility are the three most important driving forces for remanufacturing. The total sale for remanufacturing businesses in the USA before 1998 was around 53 billion dollars (Lund, 1998). Remanufacturing industry's total sales in the US went up to $\$ 43$ billion in 2011 with the expected growth of $15 \%$ in $2012^{1}$. Due to detrimental impacts of landfilling some products on soil and water resources and also animal lives, governments pass laws not only to protect the ecosystem but also to save the natural resources utilized in those products. For instance, WEEE (Waste Electrical and Electronic Equipment) directive obligates e-manufacturers to take back a proportion of e-waste (Souza, 2013). Companies also feel a social responsibility to protect the environment if no obligatory law exists. Due to the

\footnotetext{
${ }^{1}$ Remanufactured goods: An overview of the U.S. and global industries, markets, and trades, 2012
} 
reasons briefed above, some companies have decided to become involved in remanufacturing the products once they are manufactured. Xerox (Toffel, 2004) and HP (Guide Jr et al., 2008) are among the companies who have earned millions of dollar by disassembling and remanufacturing the returned products.

The reason for the existence of the potential for profit-generation is that the remanufacturer may be able to benefit from reviving parts of the production effort put into manufacturing the product in the first place; many returned products only need to be polished and packed, and the others require to be tested and repaired. Thus, remanufacturers take advantage of the energy and material inherited in the returned products. The driving forces briefed above motivate more in-depth research studies to cover different aspects of the remanufacturing industry to tackle the current and prospective challenges.

\section{$1.1 \quad$ Research Motivation}

Among the most important elements that complicate the remanufacturing process are the quality variability and uncertain timing of the returns. The quality variability leads to the processing time uncertainty. Thus, the queuing systems that cover the stochastic arrivals and processes are worth considering in the remanufacturing process modeling (Guide, 2000). One of the main reasons that complicate remanufacturing planning are the uncertain arrival of returns and processing times. Iyer and Jain (2004) study a model in which the products are classified into two categories based on their demand variability. The idea of having two different streams of returns is also investigated in 
Fathi et al. (2015), which implies that the variable quality conditions of returns and also different demand distributions can be taken into account by considering two return streams into the system. The two return streams can be interpreted as two different types of products; or even the same product with two different quality classes. For instance, computer mouses and keyboards for an electronics remanufacturer are two different types of products; computer keyboards requiring minor remanufacturing and keyboards in need of major remanufacturing are considered as two quality classes of the same product.

When the limitations of the storage capacity are taken into consideration, it can be verified that various scenarios of the storage capacity allocation to different streams of returns affect the profitability of the firm. There are two possible strategies for the capacity allocation problem. First, it can be assumed that the storage area is divided between the streams of returns. In other words, the arrival of a particular type of the returned products is blocked if the maximum capacity allocated to this product is reached. Second, it may be reasoned that merging the storage areas can result in a better utilization of the total storage capacity. Hence, instead of blocking the arrival of a particular type of the returned products once its dedicated storage area is full, the company may still accept the product and store it in the space assigned to the other streams of products. In this research, the effect of merging the storage capacity on the profitability of the company is studied as an alternative option.

Although it is widely assumed that most companies use the merged storage strategy, Fathi 
et al. (2015) mention that the divided storage strategy is a new trend for remanufacturing systems;

hence, the impact of this policy on the admission decision should be carefully investigated. However,

the number of studies that aim to mathematically model the divided or combined storage policies is

extremely limited. For instance, the merged capacity strategy for Volvo's heavy truck distribution center and Alcan Aluminum are studied in Narus and Anderson (1996) and Iyer and Jain (2004), respectively. The above discussion clarifies that accurate evaluations about which storage strategy is more often used in practice by the remanufacturers do not exist. Accordingly, this study aims at answering the following questions:

1. Should a remanufacturing company merge its storage capacity or allocate separate spaces for different products?

2. What are the factors that have an effect on this decision? Can the impact of these factors on the profitability of the selected strategy be quantified analytically?

To the best of the author's knowledge, there are no studies available in the literature that address the above research questions in a manufacturing or remanufacturing environment. As such, this paper contributes to the literature by:

- considering the deteriorating value of the remanufactured products;

- considering two $M / M / 1 / k$ production lines that divide or share a storage area; and,

- answering the question that whether merging capacity improves the performance. 
The results of this study demonstrate that selecting the correct storage policy requires not only an in-depth understanding of the models and their semantics, but also an accurate measurement of the system parameters. Furthermore, choosing the wrong storage system results in lost profits for the remanufacturers. Hence, deciding between the two studied strategies proves to be important for the companies who are active in the remanufacturing industry. The studied models are explained in detail in the following section.

\subsection{Model Description}

As mentioned before, in this paper two different storage scenarios are studied. The first strategy models the capacity allocation problem for a remanufacturing system. It is assumed that the production lines for the various remanufacturing streams are separated from each other; however, the limited storage area has to be divided between the different types of returns. It is also assumed that the remanufactured value of the products decreases over time based on a decay rate. In other words, the company is keen to process the returned items as soon as possible. Although the production lines for different remanufacturing streams are distinguished, the limited storage area has to be divided between the returns. The goal is to optimize the threshold $k$ which divides the capacity of the buffer $C$ into $C-k$ for stream 1 and $k$ for the second stream. The model for this strategy is depicted in Figure 1.

Figure 1: Please place about here.

In the second approach, the storage area is shared between the two streams of products. To 
keep the results comparable, the other assumptions of this strategy are the same as those of the first scenario. The system defined in the second strategy is depicted in Figure 2.

Figure 2: Please place about here.

For products with a short life cycle such as laptops and cell phones, the remanufacturing time impacts the value of the product. Thus, to calculate the profit, the deteriorating revenue should be considered. Furthermore, the cost of maintaining an inventory system as well as the time value of money can be implicitly regarded in the deteriorating revenue. If $r_{0}$ is the current value of the finished product, $r_{0} e^{-\beta W}$ is the deteriorating revenue of remanufacturing of the product where $\beta$ is the decay rate and $W$ is the waiting time of the product in the system (Guide Jr et al., 2008).

In this article, the goal is to maximize the company's profit by acquiring the best strategy in capacity allocation. This task is performed by developing two mathematical models. Furthermore, extensive numerical experiments are conducted and the performance of the two capacity allocation strategies are compared.

The numerical experiment evaluates and compares the behavior of the two models by changing the parameters of the models in particular intervals. Parameter values are set such that one of the products is considered to be superior compared to the other one regarding the generated profits. The numerical analysis reveals that, in the specified parameter intervals, inferior product's arrival rate has a bigger impact on the objective function than that of the superior product. On the other hand, improving the processing rate of the superior product must be given priority over making the 
processing rate of the inferior product more efficient. Moreover, to improve the company's profits, superior product's sale price is more important than the inferior product's sale price.

Although selecting between the two storage policies depends on the parameter values, valuable insight can be obtained by the numerical experiment. In the chosen parameter intervals, the merging storage system becomes more attractive as the remanufacturing cost of the superior product increases. On the contrary, the divided capacity policy becomes more appealing if the remanufacturing cost of the inferior product increases. Finally, while the elevation of the sale price of the superior product makes the divided storage policy more preferable, merged storage strategy becomes more desirable if the sale price of the inferior product increases.

\section{$2 \quad$ Literature Review}

This section starts with a discussion that highlights the differences between manufacturing

and remanufacturing systems. Afterward, capacity allocation and remanufacturing processes are

reviewed. Then, a number of papers in the area of remanufacturing inventory control are listed.

Next, the usage of queuing theory methods in remanufacturing optimization is explained. Finally,

the contributions of the paper are listed.

\subsection{Manufacturing and Remanufacturing Systems}

Major processes involved in a remanufacturing setting include product collection, sorting, inspection, routing, disassembly, cleaning, and reassembly. However, unlike conventional manu- 
facturing processes, it is almost evident that detailed steps involved in these processes, as well as their processing times, cannot be determined due to the variability in the quality of the returned products (Coyle et al., 2016). The variability in the quality of the returned products means that the processing steps and processing times of the remanufacturing processes fluctuate significantly. This is exactly the opposite of the conventional manufacturing systems in which the planner has substantial control over the manufacturing steps and the required processing times. As a result, it is often the case that the models developed for the remanufacturing system and manufacturing systems cannot be used interchangeably (Ilgin and Gupta, 2016; van der Laan, 1998). Some of the other differences between remanufacturing and manufacturing systems are summarized in table 1.

Interested reader may refer to Tibben-Lembke and Rogers (2002).

Table 1: Major differences between manufacturing and remanufacturing systems

\begin{tabular}{ccc}
\hline & Manufacturing & Remanufacturing \\
\hline Forecasting & Relatively straightforward & More difficult \\
Distribution points & One to many & Many to one \\
Product quality and packaging & Uniform & Not uniform \\
Destination and routing & Clear & Unclear \\
Pricing & Relatively uniform & Dependent on many factors \\
Costs & Easily visible & Vague and invisible \\
Inventory management & Consistent & Inconsistent \\
Product life cycle & Manageable & More complex \\
\hline
\end{tabular}

\subsection{Remanufacturing Capacity Allocation}

Natural differences between manufacturing and remanufacturing systems have inspired some researchers to either optimize pure remanufacturing systems under various assumptions, or study 
settings in which manufacturing and remanufacturing streams coexist. These studies are often categorized under titles such as "remanufacturing capacity allocation" or "remanufacturing process optimization." Kenne et al. (2012) studied a manufacturing/remanufacturing system for a single product with the objective of minimizing the sum of the holding and backlog costs. A stochastic dynamic programming model was developed and solved numerically.

Poles (2013) modeled a production and inventory system for remanufacturing. System dynamics simulation approach was employed to compare the effect of reducing the lead time as opposed to the processing time of the remanufacturing products. Li et al. (2013) presented various GERTbased models to analyze the variability of remanufacturing task sequences. Furthermore, the GERT models were employed to determine the probability of individual remanufacturing processes and the time associated with them.

Vahdani and Mohammadi (2015) developed an optimization model for designing a manufacturing/remanufacturing network with the objective of minimizing the total cost and waiting time of the products in the queues; lower bounds for the optimal solutions were discussed and the optimization model was solved by a metaheuristic approach. Wu (2015) considered a market in which both an original equipment manufacturer (OEM) and a remanufacturer compete for obtaining end-of-life (EOL) products; the equilibrium prices and the offered incentives to obtain EOL products by both the OEM and remanufacturer were obtained. 
Jiang et al. (2016) proposed a genetic algorithm which aimed to optimize the reliability and the cost of a remanufacturing system. It was assumed that the reliability differences were caused by varying qualities of the returned products; the cost structure consisted machine cost and tool cost. Shakourloo (2016) proposed a stochastic multi-objective goal programming model for the profit and cost in a remanufacturing system in which used and new products were processed together. The outputs of the goal programming model determined how much remanufacturing products must be ordered to optimize the described system. Ergo, one of the assumptions of this goal programming model was unlimited access to the remanufacturing products.

\subsection{Inventory Control in Remanufacturing}

Another stream of research in the area of remanufacturing optimization is developing an inventory management strategy for pure remanufacturing systems, or environments with both manufacturing and remanufacturing products. Inderfurth and van der Laan (2001) studied an inventory control system with both manufacturing and remanufacturing products. Their study showed that if the lead times of the two streams differ significantly, optimal control rules become very complicated; hence, the companies have to settle for sub-optimal scenarios. Takahashi et al. (2007) considered a remanufacturing system which consists of a disassembly line with two processes: reproduction and disposal; they proposed two inventory control policies for this system and compared them based on their costs. 
Chung and Wee (2011) reviewed a remanufacturing inventory system in which the product has

a rather short life cycle. They showed that the duration of life cycle, remanufacturing ratios and inventory holding costs play an important role in managing such inventory system. Hsueh (2011) considered a manufacturing and remanufacturing environment in which the products follow a certain life cycle. They derived closed form formulas for optimal production lot size, reorder point, and safety stock; these formulas lead to a different inventory control strategy for each phase of the product life cycle. Benkherouf et al. (2016) assumed an inventory system with production, remanufacturing and refurbishing activities and developed policies that minimized cost by determining the inventory level of the remanufacturing items.

\subsection{Remanufacturing and Queuing Theory}

From a different point of view, literature is rich with the efforts that model a remanufacturing process using analogies to the queuing systems. Researchers have come up with different queuing models to address different assumptions for the remanufacturing systems. $G I / G / 1$ system in Souza and Ketzenberg (2002) and $M / G / 1$ in Guide Jr et al. (2008) are two paramount instances in this context. Souza and Ketzenberg (2002) study a hybrid remanufacturing system with a two stage production line where the second stage is merged between the new and the remanufactured products. They maximize the total profit by finding an optimal mix of the new and the remanufactured products by considering an upper bound on the average order lead time. Guide Jr et al. (2008) study the disposition decision for an $M / G / 1$ system where the total deteriorated value of 
the returns should be optimized. Their work is the extension of Harrison (1975) that considers no salvage value for the returns.

Karamouzian et al. (2011) study a disposition decision in a remanufacturing system where different quality types of returns require different processes. Assuming $m$ possible stations and exponential processing times, Karamouzian et al. (2011) finds the best quantity of each product type to admit or dispose of. Later, Karamouzian et al. (2014) extended the work of Guide Jr et al. (2008) by considering the Shortest Expected Processing Time (SEPT) instead of the First Come First Served (FCFS) strategy for an $M / G / 1$ system. Maleki et al. (2017) considered a $M / M / 1 / k$ queuing system that accepts several types of nonconforming inputs and decides whether they should be processed by one of the workstations within the realm of the company, or they should be outsourced; a bi-objective mixed-integer nonlinear program was developed and solved by a genetic algorithm. Gayon et al. (2017) considered a production-inventory system in which returned products can be either disposed of or stored for remanufacturing. The system was modeled as an $M / M / 1$ make-to-stock queuing environment, and the optimal strategies were derived.

Although the literature on the queuing system applications is quite rich, capacity merging studies are not well established. Merging capacity can be performed in two forms: storage area and production line. Merging the production line capacities was studied in Fathi et al. (2015), Iyer and Jain (2004), Sahba (2012) and Yu et al. (2008). Merging the production line capacity is also referred to as capacity pooling in the literature. 
To investigate the impact of capacity pooling for a number of independent facilities, Yu et al. (2008) developed models that find the best strategy in various situations. Sahba (2012) studied the effect of capacity pooling and inventory pooling for repairable systems. Merging production line capacity for two streams of products was studied in Iyer and Jain (2004), where priority may be given to low demand variability products. Fathi et al. (2015) compared an $H+M / M / 1$ system with two separate $M / M / 1$ and $H / M / 1$ systems to investigate the impact of production line merging.

\subsection{Research Contributions}

All the research studies briefed above assume that by merging the production line capacity, the production rates will add up. However, this assumption may not hold in the remanufacturing systems due to the uncertainty of the required processes for product remanufacturing. In other words, since different processes may be required to remanufacture products with different quality conditions and since these quality conditions are unknown, merging the production lines will not result in the expected processing rate. Therefore, capacity pooling in the production stations is not practiced in this study.

This paper concentrates on the policies that control the storage area of a remanufacturing setting. The focus is on a remanufacturing environment with two distinct streams of products. Hence, a portion of the storage area can be assigned to each of these streams (divided storage policy), or the warehouse can be shared between them (merged storage approach). The divided storage policy is described by a novel nonlinear model which is stemmed from queuing theory. The 
merged storage approach is modeled using the notions of the Markovian processes. Finally, it is shown that none of the models describe a dominant storage policy; selecting the best model depends on the values of the parameters as will be explained in the forthcoming sections. Hence, a number of recommendations for selecting the best storage strategy based on the values of the parameters are given. To the best of the author's knowledge, the storage strategies and the developed models have not been addressed in the literature.

The remainder of this paper is organized as follows: detailed assumptions and mathematical models for the addressed strategies are presented in Section 3. Section 4 reports the conducted numerical experiments and compares the dedicated capacity and the merged capacity strategies. Finally, the paper is concluded by presenting a summary of this research work and directions for future research in Section 5.

\section{The Proposed Models}

Returns arrive at the system with a Poisson distribution. The company has the option of accepting or rejecting the returns. The only admission criterion is capacity; if enough capacity is available in the storage area, the returned product would be admitted. The following assumptions are made in this problem: 1) Returns arrive to the system based on a Poisson distribution. 2) Production rates for the remanufacturing lines follow a Poisson distribution. 3) There is always sufficient demand for the remanufactured products; the output of the system would always be sold. 
For the divided capacity strategy, a mathematical programming model is proposed and is called Model 1 hereinafter. Solving the model will result in the optimized allocated capacity for each stream of the returns. For the second strategy, however, a mathematical model is developed to calculate the total profit directly and will be called Model 2 hereinafter. The following notations are used throughout the remainder of this article:

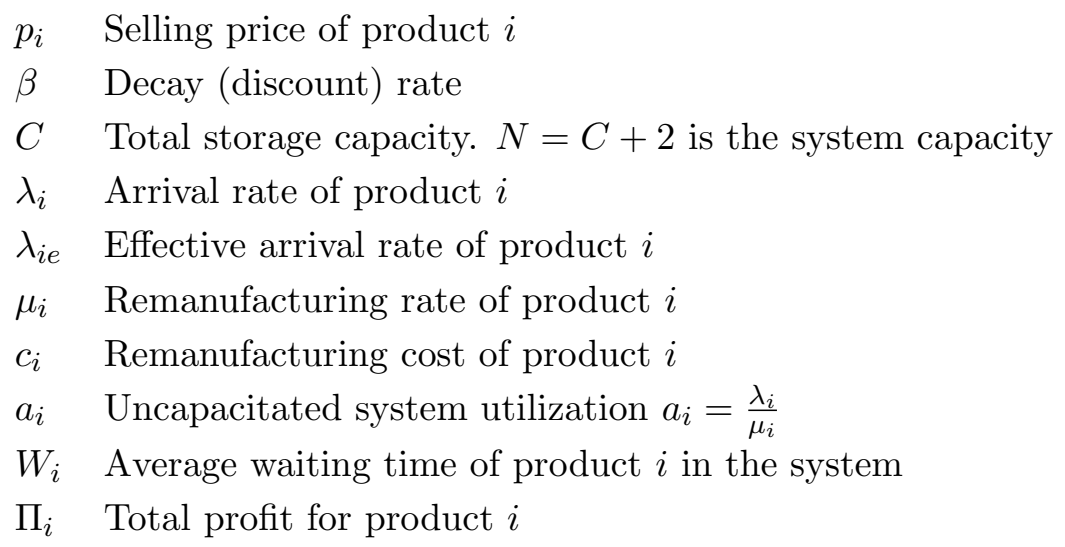

Note that the storage capacity $(C)$ represents the total storage space in the remanufacturer's warehouse. System capacity $(N)$ consists of the storage capacity of the warehouse plus the capacity of the two remanufacturing stations; this implies that each remanufacturing station can handle only one product at a time. Hence, the total system capacity is $N=C+2$. 


\subsection{Model 1}

For this strategy, each remanufacturing line could be modeled as an $M / M / 1 / k$ queuing system.

In an $M / M / 1 / k$ queuing system the expected product waiting time is:

$$
W=\frac{a\left(1-(k+1) a^{k}+k a^{k+1}\right)}{\lambda\left(1-a^{k}-a+a^{k+1}\right)}
$$

In which $a=\frac{\lambda}{\mu}$. The total number of remanufactured products in a fixed period of time, which is called the effective arrival rate, in an $M / M / 1 / k$ system is:

$$
\lambda_{e}=\lambda\left(\frac{1-a^{k}}{1-a^{k+1}}\right)
$$

For more information about $M / M / 1 / k$ queuing systems and their characteristics the reader is referred to Stidham (2009).

In this system, the capacity of the storage area is $C$ and the storage area is divided by threshold $k$. Thus, the storage capacity for the first stream is $k$ and for the second stream is $C-k$. Therefore, the system capacity for the remanufacturing lines are $k+1$ and $C-k+1$, respectively. Hence, the total remanufacturing profit can be obtained by equation (3).

$$
\Pi=\Pi_{1}+\Pi_{2}=\lambda_{1}\left(\frac{1-a_{1}^{k+1}}{1-a_{1}^{k+2}}\right)\left(p_{1} e^{-\beta W_{1}}-c_{1}\right)+\lambda_{2}\left(\frac{1-a_{2}^{C-k+1}}{1-a_{2}^{C-k+2}}\right)\left(p_{2} e^{-\beta W_{2}}-c_{2}\right)
$$

The problem is to maximize the total profit gained by remanufacturing.

$$
\text { Maximize } \Pi
$$




$$
\begin{aligned}
& W_{1}=\frac{a_{1}\left(1-(k+2) a_{1}^{k+1}+(k+1) a_{1}^{k+2}\right)}{\lambda_{1}\left(1-a_{1}^{k+1}-a_{1}+a_{1}^{k+2}\right)} \\
& W_{2}=\frac{a_{2}\left(1-(C-k+2) a_{2}^{C-k+1}+(C-k+1) a_{2}^{k+2}\right)}{\lambda_{2}\left(1-a_{2}^{C-k+1}-a_{2}+a_{2}^{C-k+2}\right)} \\
& 1 \leq k \leq C-1
\end{aligned}
$$

Equations (5) and (6) are the waiting times for the returns of products 1 and 2. Constraint (7) enforces the lower and the upper bound of the threshold $k$. This model is nonlinear and may be non-concave. However, since in practice the capacity is a finite integer number, the best threshold could be found by examining all of the possible values of the objective function (4).

\subsection{Model 2}

In the second strategy, the storage area is merged between the streams and the returns have no admission priority over one another. The system can be investigated as a Markovian process. The model states have the form of $[i, j]$, where $i$ is the number of products from the first stream in the storage area and $j$ is the quantity from the second stream. $m$ denotes the total number of states.

It is important to notice that if the system capacity is $N$, the storage area capacity is $C=N-2$. Therefore, if there are $N-1$ products from one type in the system, the system is blocked for the incoming products. It is worth noticing that only one event is possible at a time: $[i, j]$ can be 
modified to $[i \pm 1, j]$ or $[i, j \pm 1]$.

To find the performance measures for the addressed system four steps must be taken. First, the infinitesimal generator matrix $Q$ is obtained. The elements of matrix $Q$ are the transient rates from one state to another. In the second step, the steady-state probabilities should be calculated. To find the steady-state probabilities, the following linear system of equations should be solved:

$$
\begin{aligned}
& P Q=0 \\
& \sum \pi_{[i, j]}=1
\end{aligned}
$$

Where $\pi_{[i, j]}$ is the steady-state probability of state $[i, j]$ and $P$ is the $m \times m$ steady-state probability matrix. The system of equality (8) for this special case has the following form:

$$
\left\{\begin{array}{l}
\pi_{[0,0]}\left(\lambda_{1}+\lambda_{2}\right)=\pi_{[1,0]} \mu_{1}+\pi_{[0,1]} \mu_{2} \\
\pi_{[0, j]}\left(\lambda_{1}+\lambda_{2}+\mu_{2}\right)=\pi_{[0, j-1]} \lambda_{2}+\pi_{[1, j]} \mu_{1}+\pi_{[0, j+1]} \mu_{2} \quad 1 \leq j \leq C \\
\pi_{[i, 0]}\left(\lambda_{1}+\lambda_{2}+\mu_{1}\right)=\pi_{[i-1,0]} \lambda_{1}+\pi_{[i, 1]} \mu_{2}+\pi_{[i+1,0]} \mu_{1} \quad 1 \leq i \leq C \\
\pi_{[i, j]}\left(\lambda_{1}+\lambda_{2}+\mu_{1}+\mu_{2}\right)=\pi_{[i-1, j]} \lambda_{1}+\pi_{[i, j-1]} \lambda_{2}+\pi_{[i+1, j]} \mu_{1}+\pi_{[i, j+1]} \mu_{2} \quad \forall i, j \neq 0 ; i+j \leq C+1 \\
\pi_{[0, C+1]} \mu_{2}=\pi_{[1, C+1]} \mu_{1}+\pi_{[0, C]} \lambda_{2} \\
\pi_{[C+1,0]} \mu_{1}=\pi_{[C+1,1]} \mu_{2}+\pi_{[C, 0]} \lambda_{1} \\
\pi_{[1, C+1]}\left(\mu_{1}+\mu_{2}\right)=\pi_{[1, C]} \lambda_{2} \\
\pi_{[C+1,1]}\left(\mu_{1}+\mu_{2}\right)=\pi_{[C, 1]} \lambda_{1} \\
\pi_{[i, j]}\left(\mu_{1}+\mu_{2}\right)=\pi_{[i, j-1]} \lambda_{2}+\pi_{[i-1, j]} \lambda_{1} \quad \forall i, j \neq 0 ; i, j \leq C ; i+j=C+2 \\
\sum \pi_{[i, j]}=1
\end{array}\right.
$$

Solving the above system of linear equations is straightforward. However, finding the closed form for all of the steady-state probabilities is a difficult task. Next step is to find the average 
number of products from each kind in the system. The average number of products in the system could be obtained using equations (9) and (10).

$$
\begin{aligned}
& L^{(1)}=\sum_{i}\left(i \sum_{j} \pi_{[i, j]}\right) \\
& L^{(2)}=\sum_{j}\left(j \sum_{i} \pi_{[i, j]}\right)
\end{aligned}
$$

Where $L^{(1)}$ and $L^{(2)}$ are the average number of products from type 1 and 2 in the system, respectively. The final step is to find the average waiting time for each unit of each product type. Using the Little's Law for a capacitated system, the average waiting time for a product accepted by the system is:

$$
W=\frac{L}{\lambda(1-\theta)}
$$

Where $\theta$ is the blocking probability, which in the system under study is summarized by:

$$
\theta=\pi_{[0, C+1]}+\pi_{[C+1,0]}+\sum_{i+j=C+2} \pi_{[i, j]}
$$

Blocking probability is the same for both types of the products. Thus, the average unit waiting times for product type $i ; i=1,2$ could be found by equations (13).

$$
W_{i}=\frac{L^{(i)}}{\lambda_{i}(1-\theta)} ; i=1,2
$$

After obtaining the average unit waiting times, the deteriorated revenue could be calculated 
by equation (14).

$$
\Pi=\Pi_{1}+\Pi_{2}=\lambda_{1 e}\left(p_{1} e^{-\beta W_{1}}-c_{1}\right)+\lambda_{2 e}\left(p_{2} e^{-\beta W_{2}}-c_{2}\right)
$$

The effective arrival rates are calculated by equation (15).

$$
\lambda_{i e}=\lambda_{i}(1-\theta)
$$

Further information about Markovian processes and their solution procedure can be found at Ross (1996). To this end, the mathematical models for both of the studied strategies are presented.

An illustrative example will be presented in Section 3.3 to clarify the calculations of the first and the second models.

\subsection{Illustrative Example}

For the first model (divided storage area), the values presented in table 2 were used. The system capacity was set to $11(C=11)$. The best threshold $k$ could be found by graphing the value of the objective function represented by equation (3) and changing $k$ from 1 to 10 . 
Table 2: Input Data for Numerical Example

\begin{tabular}{cc}
\hline Parameter & Value \\
\hline$\mu_{1}$ & 5 per hour \\
$\mu_{2}$ & 3 per hour \\
$\lambda_{1}$ & 6 per hour \\
$\lambda_{2}$ & 1 per hour \\
$\beta$ & 0.0005 \\
$p_{1}$ & 10 \\
$p_{2}$ & 8 \\
$c_{1}$ & 5 \\
$c_{2}$ & 3 \\
\hline
\end{tabular}

Equation (3) is concave regarding the input data and the best threshold is $k=8$ with the total profit equal to 23.13 .

Figure 3: Please place about here.

Next, an example is presented for the second model. In this example, the storage area capacity is 1 . The input data are presented in table 2 . The possible set of states is:

$$
S:\{[0,0],[1,0],[1,1],[2,1],[2,0],[0,2],[1,2],[0,1]\}
$$

where $[i, j]$ denotes the number of products from type 1 and 2 in the system, respectively. As the first step, the infinitesimal generator matrix for a problem with size 3 is computed as follows: 


\begin{tabular}{|c|c|c|c|c|c|c|c|c|}
\hline & {$[0,0]$} & {$[1,0]$} & {$[1,1]$} & {$[2,1]$} & {$[2,0]$} & {$[0,2]$} & {$[1,2]$} & {$[1,0]$} \\
\hline$[0,0]$ & $-\lambda_{1}-\lambda_{2}$ & $\lambda_{1}$ & 0 & 0 & 0 & 0 & 0 & $\lambda_{2}$ \\
\hline$[1,0]$ & $\mu_{1}$ & $-\lambda_{1}-\lambda_{2}-\mu_{1}$ & $\lambda_{2}$ & 0 & $\lambda_{1}$ & 0 & 0 & 0 \\
\hline$[1,1]$ & 0 & $\mu_{2}$ & $-\lambda_{1}-\lambda_{2}-\mu_{1}-\mu_{2}$ & $\lambda_{1}$ & 0 & 0 & $\lambda_{2}$ & $\mu_{1}$ \\
\hline$[2,1]$ & 0 & 0 & $\mu_{1}$ & $-\mu_{1}-\mu_{2}$ & $\mu_{2}$ & 0 & 0 & 0 \\
\hline$[2,0]$ & 0 & $\mu_{1}$ & 0 & 0 & $-\mu_{1}$ & 0 & 0 & 0 \\
\hline$[0,2]$ & 0 & 0 & 0 & 0 & 0 & $-\mu_{2}$ & 0 & $\mu_{2}$ \\
\hline$[1,2]$ & 0 & 0 & $\mu_{2}$ & 0 & 0 & $\mu_{1}$ & $-\mu_{1}-\mu_{2}$ & 0 \\
\hline$[0,1]$ & $\mu_{2}$ & 0 & $\lambda_{1}$ & 0 & 0 & $\lambda_{2}$ & 0 & $-\lambda_{1}-\lambda_{2}-\mu_{2}$ \\
\hline
\end{tabular}

Replacing the parameters with the values presented in table 2 yields:

\begin{tabular}{|c|c|c|c|c|c|c|c|c|}
\hline \multicolumn{9}{|c|}{$[0,0][1,0][1,1][2,1][2,0][0,2][1,2][0,1]$} \\
\hline$[0,0]$ & -7 & 6 & 0 & 0 & 0 & 0 & 0 & 1 \\
\hline$[1,0]$ & 5 & -12 & 1 & 0 & 6 & 0 & 0 & 0 \\
\hline$[1,1]$ & 0 & 3 & -15 & 6 & 0 & 0 & 1 & 5 \\
\hline$[2,1]$ & 0 & 0 & 5 & -8 & 3 & 0 & 0 & 0 \\
\hline$[2,0]$ & 0 & 5 & 0 & 0 & -5 & 0 & 0 & 0 \\
\hline$[0,2]$ & 0 & 0 & 0 & 0 & 0 & -3 & 0 & 3 \\
\hline$[1,2]$ & 0 & 0 & 3 & 0 & 0 & 5 & -8 & 0 \\
\hline$[0,1]$ & 3 & 0 & 6 & 0 & 0 & 1 & 0 & -10 \\
\hline
\end{tabular}

Using the pattern obtained for system of equality (8), the following system of linear equalities 
are required to be solved for the second step:

$$
\left\{\begin{array}{l}
\pi_{[0,0]}(6+1)=5 \pi_{[1,0]}+3 \pi_{[0,1]} \\
\pi_{[0,1]}(6+1+3)=\pi_{[0,0]}+5 \pi_{[1,1]}+3 \pi_{[0,2]} \\
\pi_{[1,0]}(6+1+5)=6 \pi_{[0,0]}+3 \pi_{[1,1]}+5 \pi_{[2,0]} \\
\pi_{[1,1]}(6+1+5+3)=6 \pi_{[0,1]}+\pi_{[1,0]}+5 \pi_{[2,1]}+3 \pi_{[1,2]} \\
\pi_{[0,2]}(3)=5 \pi_{[1,2]}+\pi_{[0,1]} \\
\pi_{[2,0]}(5)=3 \pi_{[2,1]}+6 \pi_{[1,0]} \\
\pi_{[1,2]}(5+3)=\pi_{[1,1]} \\
\pi_{[2,1]}(5+3)=6 \pi_{[1,1]} \\
\sum_{i, j} \pi_{[i, j]}=1
\end{array}\right.
$$

Solving the above system results in the values reported in table 3 .

Table 3: Steady-state probabilities for the numerical example

\begin{tabular}{ccccccccc}
\hline State & {$[0,0]$} & {$[1,0]$} & {$[1,1]$} & {$[2,1]$} & {$[2,0]$} & {$[0,2]$} & {$[1,2]$} & {$[0,1]$} \\
\hline Probability & 0.21 & 0.259 & 0.056 & 0.042 & 0.336 & 0.031 & 0.007 & 0.058 \\
\hline
\end{tabular}

The third step is to find the average number of jobs in the system for both types. Using equations (9) and (10), $L^{(1)}=1.078$ and $L^{(2)}=0.232$ are obtained. The blocking probability $\theta$ could be calculated by equation (12). Having the value of $\theta(0.416)$ and using equation (13), the average waiting times for products 1 and 2 would be 18.46 and 23.84 minutes, respectively. These values were verified by a simulation model with an error margin of 0.01 . The simulation model was created and run for 20,000 hours with 2,000 hours as the warm-up period. 


\section{$4 \quad$ Numerical Experiments}

In this section, a factor analysis on the mathematical models are carried out. The input value for the numerical experiments are presented in table 4.

Table 4: Input values for the numerical experiments

\begin{tabular}{cc}
\hline Parameter & Values \\
\hline$p_{1}$ & $10,20,30$ \\
$p_{2}$ & $30,50,70$ \\
$\beta$ & $0.001,0.003,0.005$ \\
$\lambda_{1}$ & $30,60,90$ \\
$\lambda_{2}$ & $30,60,90$ \\
$\mu_{1}$ & $15,35,55$ \\
$\mu_{2}$ & $5,15,25$ \\
$c_{1}$ & $5,10,15$ \\
$c_{2}$ & $10,15,25$ \\
$C$ & 30 \\
\hline
\end{tabular}

All parameters have three levels each. Here, it is assumed that the product with higher processing time and higher cost yields more profit. Therefore, product type 2 is the superior product regarding the sale value. The deterioration rate has a broad range to capture the perishability of the outputs. Only one value is assigned to the capacity as the higher the capacity is, the less impact it has on the optimum strategy. It is worth mentioning that, in practice, the remanufacturer can have an impact (however limited it may be) on the arrival rates of the products. For instance, increasing the arrival rates can be done by augmenting the marketing exertion; decreasing the arrival rates is possible by refusing to accept some of the arrivals. To trace the changes they cause on the objective functions and the optimum decisions, a wide range of variability was allotted to the parameters. 
Two independent sets of numerical experiments were conducted in this article. The first one is devoted to finding the overall relation between each storage strategy, its associated total profit and model parameters and will be described in section 4.1. In section 4.2, the storage strategies are compared and the conditions for which each of them outperforms the other one is discussed.

It should be noted that the obtained results and conclusions in sections 4.1 and 4.2 are extracted based on the numerical analysis as outlined above. Hence, the results and conclusions are valid within the intervals defined in table 4 , and cannot be generalized to other parameter values.

Extracting analytical results from the mathematical formulas would have been much more effective than the above approach. However, the complexity of the models (both in the divided and the merged storage policies), make this almost impossible. As a result, in practice, in order for the developed models to be beneficial for a remanufacturer, parameter values should be measured for the environment in which the remanufacturer operates; and similar numerical analysis must be performed. The following numerical method can be employed as a template for similar experiments by the remanufacturers.

\subsection{Factor Analysis}

It is worth mentioning that in this numerical experiment, the numbers do not bear importance;

the engaging matter is the change direction. Such analysis empowers one to answer imperative questions such as whether increasing $c_{2}$ results in higher profit changes in the divided storage area 
or the merged warehousing strategy. Using the data presented in table 4 as the input for the divided storage strategy leads in the following results:

Table 5: Factor analysis for the divided storage strategy

\begin{tabular}{cccccccccc}
\hline Parameters & $p_{1}$ & $p_{2}$ & $c_{1}$ & $c_{2}$ & $\lambda_{1}$ & $\lambda_{2}$ & $\mu_{1}$ & $\mu_{2}$ & $\beta$ \\
\hline Level 1 & 453 & 499 & 919 & 851 & 697 & 715 & 595 & 484 & 764 \\
Level 2 & 761 & 763 & 761 & 785 & 796 & 778 & 780 & 780 & 763 \\
Level 3 & 1077 & 1029 & 610 & 653 & 797 & 796 & 915 & 1026 & 762 \\
\hline Overall Impact & $\uparrow$ & $\uparrow$ & $\downarrow$ & $\downarrow$ & $\uparrow$ & $\uparrow$ & $\uparrow$ & $\uparrow$ & $\downarrow$ \\
\hline
\end{tabular}

The values reported in table 5 are the total profit values. The model for the divided storage area is solved for the same data as the merged storage area. However, the results are not quite the same. The impact of the inferior product's sale price is stronger than the superior product's sale price. Moreover, increasing the arrival rate of both of the products results in higher total profits, which is different for the merged storage area case. The following conclusions could be made from the results reported in table 5 . Note that these conclusions are only valid in the given parameter intervals.

- The objective function is non-linear around all the parameters (for $\beta$, since the change is insignificant the non-linearity can be overlooked).

- The impact of the inferior product's remanufacturing cost is more severe than that of the superior product.

- The effect of the inferior product's arrival rate is more severe than that of the superior product.

- The effect of the inferior product's processing rate is less severe than that of the superior 
product.

- Although increasing the arrival rate yields in higher profits, there is a threshold after which there is a weak and insignificant chance of increasing the returns since the storage area is full; and consequently increasing the arrival rate cannot endlessly result in the profit improvement.

The same analysis could be conducted for the merged storage area strategy. The results for this policy, based on the values prescribed in table 4, are as follows:

Table 6: Factor analysis for the merged storage strategy

\begin{tabular}{cccccccccc}
\hline Parameters & $p_{1}$ & $p_{2}$ & $c_{1}$ & $c_{2}$ & $\lambda_{1}$ & $\lambda_{2}$ & $\mu_{1}$ & $\mu_{2}$ & $\beta$ \\
\hline Level 1 & 484 & 335 & 709 & 746 & 559 & 780 & 608 & 209 & 636 \\
Level 2 & 634 & 634 & 634 & 671 & 634 & 634 & 634 & 634 & 634 \\
Level 3 & 784 & 932 & 559 & 521 & 709 & 584 & 636 & 1059 & 631 \\
\hline Overall Impact & $\uparrow$ & $\uparrow$ & $\downarrow$ & $\downarrow$ & $\uparrow$ & $\downarrow$ & $\uparrow$ & $\uparrow$ & $\downarrow$ \\
\hline
\end{tabular}

As expected, the faster the products are processed, the more is the firm's acquired profit. Additionally, more expensive final products and less costly remanufacturing process result in an increase in the total profit. However, the reverse impact of the superior product's arrival rate on the total profit is counter intuitive. The superior product has a higher sale price, is being processed slower and has a higher remanufacturing cost. The possible explanation for this reverse impact is as follows: although the superior product has a higher sale price, the slower remanufacturing rate results in congestion in the storage area; moreover, the higher remanufacturing cost of the superior product nullifies the potential profit created from its higher sale price. Alternatively, the inferior product can be remanufactured faster and with a lower cost. Moreover, the impact of $\beta$ for the 
given values is fairly small on the total profit.

In addition to the direct effects, comparing the impact of the parameters on the total profit is interesting. This can be obtained by changing the parameter values and observing the impact of the change on the total profit values as demonstrated by table 6. Results (valid within the considered parameter intervals) are as follows:

- The impact of the price of the superior product is more significant than the inferior product's price.

- The impact of the superior product's processing rate is more significant than the inferior product's processing rate.

- All the parameters have a non-linear effect on the total profit.

In the next section, the two strategies are compared regarding the optimal profits.

\subsection{Strategy Comparison}

This section is devoted to comparing the aforementioned strategies in terms of profitability.

For this numerical analysis, two levels (high and low) for each parameter were employed. For each combination of the parameters, the objective function values were compared to identify the conditions at which one strategy outperforms the other one. Since the objective function is not significantly sensitive to changes in the value of $\beta$, this parameter was removed from the set of 
variables whose changes may affect the optimal profit. Table 7 contains the parameter values used for the numerical experiments in this section. The high values are the largest values presented in table 4 and the low values are the smallest values in that table.

Table 7: The parameter values for the numerical experiments in section 4.2

\begin{tabular}{ccc}
\hline Parameter & Low Value & High Value \\
\hline$p_{1}$ & 10 & 30 \\
$p_{2}$ & 30 & 70 \\
$\lambda_{1}$ & 30 & 90 \\
$\lambda_{2}$ & 30 & 90 \\
$\mu_{1}$ & 15 & 55 \\
$\mu_{2}$ & 5 & 25 \\
$c_{1}$ & 5 & 15 \\
$c_{2}$ & 10 & 25 \\
$\beta$ & 0.001 & 0.005 \\
\hline
\end{tabular}

To investigate the impact of capacity on profitability, two values were considered for the capacity of the storage area. Initially, 16 cases for the combination of arrival and processing rates were studied. The reason for selecting these parameters is that they are non-commercial factors which may not be directly controllable by the remanufacturer. Noted that the remanufacturer can still have an indirect impact on these factors. For instance, increasing the arrival rate can be done by augmenting the marketing budget; decreasing the arrival rate is possible by refusing to accept some of the arrivals. All the other values were fixed at their average levels. Table 8 reports the optimal profits for each case and each strategy. 
Table 8: Impact of storage strategy, arrival and process rates on profitability

\begin{tabular}{cccccccc}
\hline Combination & \multicolumn{2}{c}{ Levels } & & $\begin{array}{c}\text { Profit for } \\
\text { Divided } \\
\text { Capacity }\end{array}$ & $\begin{array}{c}\text { Profit for } \\
\text { Merged } \\
\text { Capacity }\end{array}$ & $\begin{array}{c}\text { Absolute } \\
\text { Difference }\end{array}$ \\
& $\lambda_{1}$ & $\lambda_{2}$ & $\mu_{1}$ & $\mu_{2}$ & & & \\
\hline 1 & High & High & High & High & 1372 & 1123 & 249 \\
2 & High & High & High & Low & 722 & 223 & 499 \\
3 & High & High & Low & High & 974 & 673 & 301 \\
4 & High & High & Low & Low & 323 & 223 & 100 \\
5 & High & Low & High & High & 1182 & 1171 & 11 \\
6 & High & Low & High & Low & 718 & 323 & 395 \\
7 & High & Low & Low & High & 784 & 324 & 460 \\
8 & High & Low & Low & Low & 320 & 296 & 24 \\
9 & Low & High & High & High & 1124 & 956 & 168 \\
10 & Low & High & High & Low & 474 & 190 & 284 \\
11 & Low & High & Low & High & 974 & 956 & 18 \\
12 & Low & High & Low & Low & 323 & 190 & 133 \\
13 & Low & Low & High & High & 934 & $1092^{*}$ & $158^{*}$ \\
14 & Low & Low & High & Low & 470 & 223 & 247 \\
15 & Low & Low & Low & High & 784 & 672 & 112 \\
16 & Low & Low & Low & Low & 320 & 223 & 97 \\
\hline
\end{tabular}

* Merged capacity policy yields are better than divided capacity yields.

All the reported objective function values in Table 7 are rounded. Among the numerical scenarios defined in this table, the only case the merging capacity strategy yields better results than the divided storage area is identified by the asterisk sign. In this particular scenario, the arrival rates are low, and the processing rates are high. Thus, one important conclusion from comparing the results is that the superiority of merging capacity strategy is for the cases in which the arrival and the processing rates are close to each other. In this scenario, the storage area undergoes less congestion, which leads to less rejection of the returns due to the storage space being full. The same conclusion could be made by inspecting the absolute differences in table 8 . 
For instance, in the High-Low-High-High scenario, the processing rates are increasing while one of the arrival rates is low. Therefore, the difference in the optimal profits is about $1 \%$, which is infinitesimal.

Where the arrival and processing rates are at the extreme ends in such way that the arrival rate is higher, the difference of the optimal profit values is relatively large. Moreover, comparing these scenarios reveals that the impact of changing one parameter on the absolute optimum value differences depends on the value of the other parameters as well. For example, the only difference between scenarios 1 and 2, and also 3 and 4 is the value of $\mu_{2}$. However, in one case decreasing $\mu_{2}$ increases the optimum profit difference, and in the other case it reduces the optimum value difference. Thus, knowing the values of the arrival and processing rates is crucial in deciding upon the best storage strategy.

Now that the impact of non-commercial factors on the best strategy choice is investigated, commercial factors can be studied. Table 8 contains the optimum profit values for commercial factors for the two strategies.

Inspecting the data reported in table 9 reveals interesting results about the storage strategies. There are four scenarios in which the merged capacity approach outperforms the divided storage strategy, and in all of them, the price of the superior product is at the low level. The following observations are made from the reported results. As usual, these results are valid within the considered parameter intervals. 
Table 9: Impact of storage strategy, sale price and remanufacturing costs on profitability

\begin{tabular}{cccccccc}
\hline Combination & & Levels & & $\begin{array}{c}\text { Profit for } \\
\text { Divided } \\
\text { Capacity }\end{array}$ & $\begin{array}{c}\text { Profit for } \\
\text { Merged } \\
\text { Capacity }\end{array}$ & $\begin{array}{c}\text { Absolute } \\
\text { Difference }\end{array}$ \\
& $p_{1}$ & $p_{2}$ & $c_{1}$ & $c_{2}$ & & & \\
\hline 1 & High & High & High & High & 1166 & 897 & 269 \\
2 & High & High & High & Low & 1380 & 1122 & 258 \\
3 & High & High & Low & High & 1515 & 1047 & 468 \\
4 & High & High & Low & Low & 1729 & 1272 & 457 \\
5 & High & Low & High & High & 594 & 299 & 295 \\
6 & High & Low & High & Low & 809 & 524 & 285 \\
7 & High & Low & Low & High & 943 & 449 & 494 \\
8 & High & Low & Low & Low & 1158 & 674 & 484 \\
9 & Low & High & High & High & 467 & $597^{*}$ & $130^{*}$ \\
10 & Low & High & High & Low & 681 & $822^{*}$ & $141^{*}$ \\
11 & Low & High & Low & High & 816 & 747 & 69 \\
12 & Low & High & Low & Low & 1031 & 972 & 59 \\
13 & Low & Low & High & High & -103 & $-1^{*}$ & $102^{*}$ \\
14 & Low & Low & High & Low & 110 & $224^{*}$ & $114^{*}$ \\
15 & Low & Low & Low & High & 245 & 149 & 96 \\
16 & Low & Low & Low & Low & 459 & 374 & 85 \\
\hline
\end{tabular}

* Merged capacity policy yields are better than divided capacity yields. 
- Increasing the remanufacturing cost of the superior product makes the merging capacity strategy more preferable.

- Increasing the remanufacturing cost of the inferior product makes the divided capacity strategy more preferable.

- Increasing the sale price of the superior product makes the divided capacity strategy more preferable.

- Increasing the sale price of the inferior product makes the merged capacity strategy more preferable.

Figure 4 illustrates the effect of changing the model parameters on the total profit values of the two storage policies. This figure is in agreement with the above results. Thus, the overall conclusion from table 9 is that the remanufacturing cost and the sale price of the products could have opposite impacts on the best storage strategy depending on the remanufacturing cost and the sale price of the other product.

Figure 4: Please place about here.

The final test is to investigate the effect of the storage capacity on the total profit and the preferred strategy. Among the 32 scenarios defined in tables 8 and 9, two scenarios are selected such that in one of them the divided storage policy is superior and in the other one the merged storage strategy performs better. Then the capacity is modified to check whether it has any impact on 
the preferred storage strategy. By this test, it is assumed that the two scenarios are representative of all the other cases. In other words, it is assumed that the interaction between the capacity value and the value of the model parameters does not have a behavioral impact on the best storage strategy. This assumption is valid as the capacity value only changes the scale of the impacts, not the impacts themselves. Scenarios 9 and 13 are selected from table 8 as these two scenarios have almost the same amount of difference in the optimum profit. In table 10, scenario 9 of table 8 is investigated; in this scenario the divided strategy performed better.

Table 10: Impact of capacity on profit for scenario 9 in table 8

\begin{tabular}{ccc}
\hline Storage Capacity $(C)$ & Divided Capacity Profit & Merged Capacity Profit \\
\hline 3 & 1100.59 & 952.51 \\
4 & 1112.17 & 956.18 \\
5 & 1118.09 & 957.08 \\
6 & 1121.2 & 957.22 \\
7 & 1122.87 & 957.15 \\
8 & 1123.77 & 957.02 \\
9 & 1124.26 & 956.88 \\
10 & 1124.52 & 956.73 \\
15 & 1124.83 & 955.98 \\
20 & 1124.84 & 955.23 \\
25 & 1124.84 & 954.49 \\
30 & 1124.84 & 953.74 \\
35 & 1124.84 & 952.99 \\
40 & 1124.84 & 952.25 \\
45 & 1124.84 & 951.5 \\
50 & 1124.84 & 950.75 \\
\hline
\end{tabular}

Two main observations can be made from the data presented in table 10. The first observation is that for the divided storage strategy, at first, increasing $C$ results in an increase in the objective 
function, but after a threshold increasing its value has no impact on the total profit. Thus, increasing the storage area capacity is not strictly beneficial if the storage strategy is to divide the storage area between the streams. For the merged strategy, however, the impact is different. At first increasing the storage area yields in higher profits. However, after a certain point $(C=6)$, the effect is reversed, and the total profit decreases. One possible explanation is that the firm accepts the returns if there is free space in the storage area. Increasing the storage capacity means admitting more returns and a longer queue. However, because of the impact of the deterioration rate, it may not be profitable to accept more returns and create longer queues. Under the divided storage capacity, this particular scenario does not generate the same results. The second observation is that changing the storage capacity does not alter the better storage policy. Figure 5 visualizes this effect.

Figure 5: Please place about here.

The same test is conducted on scenario 13 of table 10, where the merged strategy outperforms the divided approach. Results are presented in table 11. 
Table 11: Impact of capacity on profit for scenario 13 in table 8

\begin{tabular}{ccc}
\hline Storage Capacity $(C)$ & Divided Capacity Profit & Merged Capacity Profit \\
\hline 3 & 910.11 & 949.18 \\
4 & 921.7 & 994.71 \\
5 & 927.62 & 1025.57 \\
6 & 930.73 & 1047.49 \\
7 & 932.4 & 1063.6 \\
8 & 933.3 & 1075.75 \\
9 & 933.79 & 1085.08 \\
10 & 934.05 & 1092.35 \\
15 & 934.35 & 1111.54 \\
20 & 934.36 & 1117.98 \\
25 & 934.37 & 1120.02 \\
30 & 934.37 & 1120.37 \\
35 & 934.37 & 1120.06 \\
40 & 934.37 & 1119.49 \\
45 & 934.37 & 1118.82 \\
50 & 934.37 & 1118.1 \\
\hline
\end{tabular}

Table 11 shows the same behavior as table 10 for each strategy. For the divided plan, increasing the capacity results in increasing the total profit at the beginning, but after a certain threshold, it does not show a significant impact on the objective function value. Moreover, in the merged strategy, the total profit increases at first when the capacity increases, but starts to decrease after a certain capacity is reached. In other words, capacity optimization becomes an important issue if the merged strategy is adopted. Numbers in table 11 are visualized in figure 6 .

Figure 6: Please place about here.

Tables 10 and 11 demonstrate that although the change in the storage capacity has an impact on the total profit, it does not alter the preferred storage strategy. Table 12 demonstrates the 
impact of the decay rate in selecting the optimal storage policy. This table demonstrates that the

effect of the decay rate on the merged storage strategy is more prominent than the divided storage

policy. Furthermore, in both levels of the decay rate, divided storage policy dominates the merged

capacity strategy. Hence, the impact of the decay rate in the considered parameter intervals makes

the divided capacity policy more attractive.

Table 12: Impact of decay rate on profitability

\begin{tabular}{ccccc}
\hline Combination & $\begin{array}{c}\text { Level of the } \\
\text { Decay Rate } \\
(\beta)\end{array}$ & $\begin{array}{c}\text { Profit for } \\
\text { Divided } \\
\text { Capacity }\end{array}$ & $\begin{array}{c}\text { Profit for } \\
\text { Merged } \\
\text { Capacity }\end{array}$ & $\begin{array}{c}\text { Absolute } \\
\text { Difference }\end{array}$ \\
\hline 1 & Low & 836.827 & 636.472 & 200.355 \\
2 & High & 836.804 & 632.377 & 204.427 \\
\hline
\end{tabular}

In the next section, the contributions of this article are summarized and the conclusions from the numerical experiments are stated.

\section{Conclusions}

In this paper, two possible storage strategies for two streams of returns in a remanufacturing company were modeled. This problem could also be seen as a production system where the firm produces two products, but the storage area is limited. In the latter case, the streams of returns could be translated to demands. The two storage strategies compared are: 1) Divided storage strategy in which the fixed capacity of the storage area is divided into two sections and each stream has its dedicated predefined area. 2) Merged storage policy, where the entire storage area is shared between the two streams without allocating a fixed capacity to the streams of products. 
Comparing these two strategies is interesting from an operational point of view as the two products are different in terms of the remanufacturing cost, sale price, arrival rate, and processing rate. On the one hand, it is not clear in advance if merging the storage areas results in more profit; it is probable that the inferior product fills the storage space, which results in the rejection of the returns associated with the product with a higher sale price. On the other hand, dividing the storage area may reduce the system efficiency. It is possible that one stream of product is rejected because the devoted storage area is full, while there is empty space in the area allocated to the other product. Thus, modeling and comparing the two strategies is very crucial.

To analyze the system, the divided storage strategy was modeled using a mathematical model, while the merged storage strategy was described using queuing theory. Since finding the closed form for the optimal profit is impossible, numerical analysis was conducted to investigate the performance of the two strategies as well as the impact of the model parameters on the total profit values.

It was shown that when the storage area is merged, increasing the arrival rates does not necessarily result in increasing the profits. However, for the case of the divided storage, increasing the arrival rates results in more profits. Moreover, it was observed that the superiority of one strategy over the other one depends on the model parameters such as the processing rates and the sale prices.

It was assumed that one product is superior in terms of the sale price, but it has higher remanufacturing cost and also lower processing rate. It was concluded that preference of one strategy over 
the other one depends on the type of product, in addition to the parameter values. For example, while increasing the sale price of the inferior product makes the merged capacity strategy more preferable, increasing the sale price of the superior product makes the divided capacity approach more desirable. Hence, one strategy cannot be prescribed for all cases.

Finally, the numerical analysis revealed that if the storage space is divided, increasing the capacity up to a certain level is beneficial; afterward, increasing the storage capacity is not recommended. For the merged capacity policy, there is one optimum point for the storage capacity because the objective function is concave with regards to the storage area capacity.

In this research, the unconstrained problem was modeled for the two strategies. It is possible that technical limitations such as required storage conditions significantly alter the conclusions. This results in constrained models. Study of the constrained model for the case of the merged capacity is mathematically complicated but makes a framework for extending the model to more realistic and general cases.

The developed models in this research consider two streams of the returned products, i.e., two different types of returned goods or one returned product with two different quality classes. It would be interesting to generalize the models such that they accept more than two streams of the returned products. Generalizing the models is mathematically interesting, yet very challenging. Moreover, including the quality specifications of the returned products as a parameter into the developed models is quite motivating for future research efforts. 
Also, further numerical analysis is valuable from a practical point of view. It was observed that the model parameters do not relate linearly to the total profit. Thus, there may be a threshold after which the impact of the parameters is negligible. Investigating and comparing the two strategies in these situations could be an interesting course for the practitioners. Finally, extending the production lines to multi-stage prototypes is of interest from the practical and also the mathematical point of view.

\section{Acknowledgment}

The author is grateful to Dr. Mostafa Pazoki of HEC Montréal for his invaluable contributions in analyzing the models of this study. Furthermore, the author is thankful to the anonymous reviewers for their precious comments that resulted in significant improvements in the presentation of the article.

\section{References}

Benkherouf, L., Skouri, K., and Konstantaras, I. (2016). Optimal control of production, remanufacturing and refurbishing activities in a finite planning horizon inventory system. Journal of Optimization Theory and Applications, 168(2):677-698.

Chung, C.-J. and Wee, H.-M. (2011). Short life-cycle deteriorating product remanufacturing in a green supply chain inventory control system. International Journal of Production Economics, 129(1):195-203. 
Coyle, J., Langley, C., Novack, R., and Gibson, B. (2016). Supply Chain Management: A Logistics Perspective. Cengage Learning.

Fathi, M., Zandi, F., and Jouini, O. (2015). Modeling the merging capacity for two streams of product returns in remanufacturing systems. Journal of Manufacturing Systems, 37:265-276.

Gayon, J.-P., Vercraene, S., and Flapper, S. D. P. (2017). Optimal control of a production-inventory system with product returns and two disposal options. European Journal of Operational Research.

Guide, V. D. R. (2000). Production planning and control for remanufacturing: industry practice and research needs. Journal of operations Management, 18(4):467-483.

Guide Jr, V. D. R., Gunes, E. D., Souza, G. C., and Van Wassenhove, L. N. (2008). The optimal disposition decision for product returns. Operations Management Research, 1(1):6-14.

Harrison, J. M. (1975). Dynamic scheduling of a multiclass queue: Discount optimality. Operations Research, 23(2):270-282.

Hsueh, C.-F. (2011). An inventory control model with consideration of remanufacturing and product life cycle. International Journal of Production Economics, 133(2):645-652.

Ilgin, M. and Gupta, S. (2016). Remanufacturing Modeling and Analysis. CRC Press.

Inderfurth, K. and van der Laan, E. (2001). Leadtime effects and policy improvement for stochastic inventory control with remanufacturing. International Journal of Production Economics, $71(1): 381-390$. 
Iyer, A. V. and Jain, A. (2004). Modeling the impact of merging capacity in production-inventory systems. Management Science, 50(8):1082-1094.

Jiang, Z., Zhou, T., Zhang, H., Wang, Y., Cao, H., and Tian, G. (2016). Reliability and cost optimization for remanufacturing process planning. Journal of Cleaner Production, 135:16021610.

Karamouzian, A., Naini, S. G. J., and Mazdeh, M. M. (2014). Management of returned products to a remanufacturing facility considering arrival uncertainty and priority processing. International Journal of Operational Research, 20(3):331-340.

Karamouzian, A., Teimoury, E., and Modarres, M. (2011). A model for admission control of returned products in a remanufacturing facility using queuing theory. The International Journal of Advanced Manufacturing Technology, 54(1-4):403-412.

Kenne, J.-P., Dejax, P., and Gharbi, A. (2012). Production planning of a hybrid manufacturingremanufacturing system under uncertainty within a closed-loop supply chain. International Journal of Production Economics, 135(1):81-93.

Li, C., Tang, Y., Li, C., and Li, L. (2013). A modeling approach to analyze variability of remanufacturing process routing. IEEE Transactions on Automation Science and Engineering, 10(1):86-98. 
Lund, R. (1998). Remanufacturing: an american resource. In Proceedings of the Fifth International Congress Environmentally Conscious Design and Manufacturing, volume 16. June.

Maleki, L., Pasandideh, S. H. R., Niaki, S. T. A., and Cárdenas-Barrón, L. E. (2017). Determining the prices of remanufactured products, capacity of internal workstations and the contracting strategy within queuing framework. Applied Soft Computing, 54:313-321.

Narus, J. A. and Anderson, J. C. (1996). Rethinking distribution: adaptive channels. Harvard Business Review, 74(4):112.

Poles, R. (2013). System dynamics modelling of a production and inventory system for remanufacturing to evaluate system improvement strategies. International Journal of Production Economics, 144(1):189-199.

Ross, S. (1996). Stochastic processes. Wiley series in probability and statistics: Probability and statistics. Wiley.

Sahba, P. (2012). The Benefit of Capacity Pooling for Repairable Spare Parts. PhD thesis, University of Toronto.

Shakourloo, A. (2016). A multi-objective stochastic goal programming model for more efficient remanufacturing process. The International Journal of Advanced Manufacturing Technology, pages $1-15$. 
Souza, G. C. (2013). Closed-loop supply chains: A critical review, and future research. Decision Sciences, 44(1):7-38.

Souza, G. C. and Ketzenberg, M. (2002). Two-stage make-to-order remanufacturing with servicelevel constraints. International Journal of Production Research, 40(2):477-493.

Stidham, S. (2009). Optimal Design of Queueing Systems. CRC Press.

Takahashi, K., Morikawa, K., Takeda, D., Mizuno, A., et al. (2007). Inventory control for a markovian remanufacturing system with stochastic decomposition process. International Journal of Production Economics, 108(1):416-425.

Tibben-Lembke, R. S. and Rogers, D. S. (2002). Differences between forward and reverse logistics in a retail environment. Supply Chain Management: An International Journal, 7(5):271-282.

Toffel, M. W. (2004). Strategie management of product recovery. California management review, 46(2):120-141.

Vahdani, B. and Mohammadi, M. (2015). A bi-objective interval-stochastic robust optimization model for designing closed loop supply chain network with multi-priority queuing system. International Journal of Production Economics, 170:67-87.

van der Laan, E. (1998). Inventory control for joint manufacturing and remanufacturing. INSEAD working paper. INSEAD. 
Wu, C.-H. (2015). Strategic and operational decisions under sales competition and collection competition for end-of-use products in remanufacturing. International Journal of Production Economics, 169:11-20.

Yu, Y., Benjaafar, S., and Gerchak, Y. (2008). Capacity pooling and cost sharing among independent firms in the presence of congestion. Under Review. 

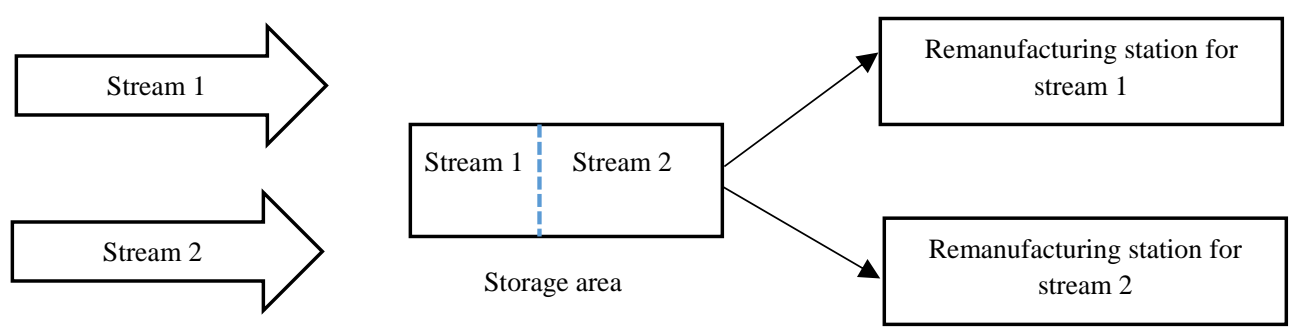

Figure 1: Illustration of the dedicated capacity model

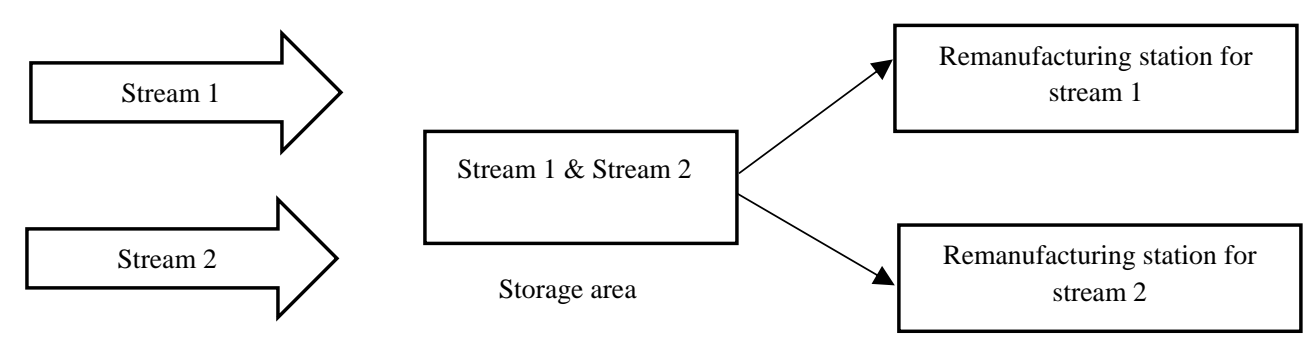

Figure 2: Illustration of the merged capacity model

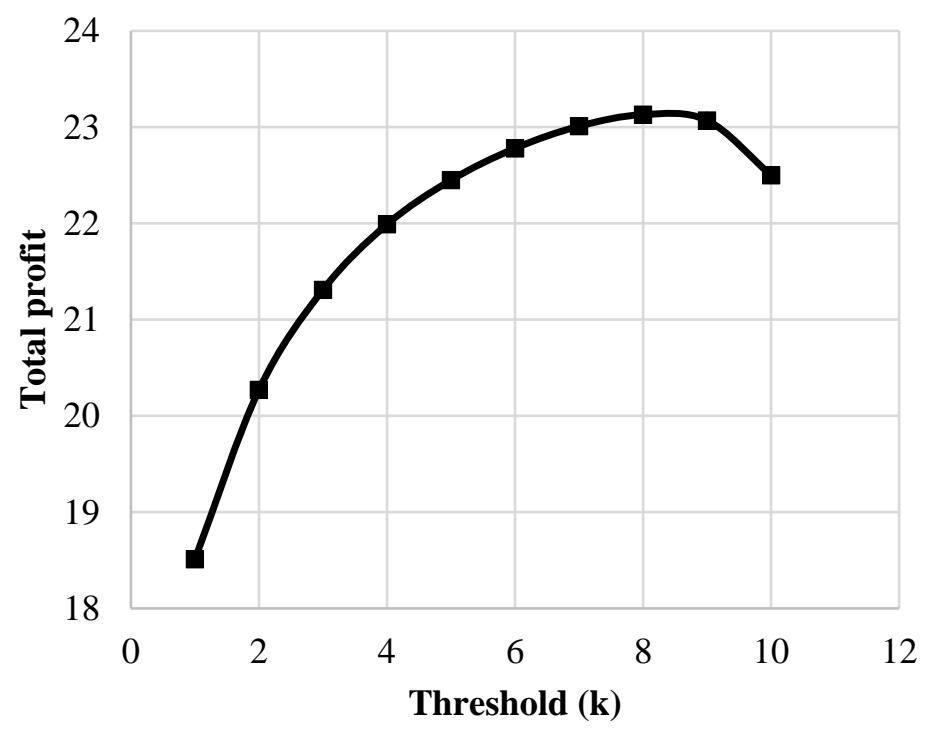

Figure 3: Profit-threshold graph for the divided storage area 


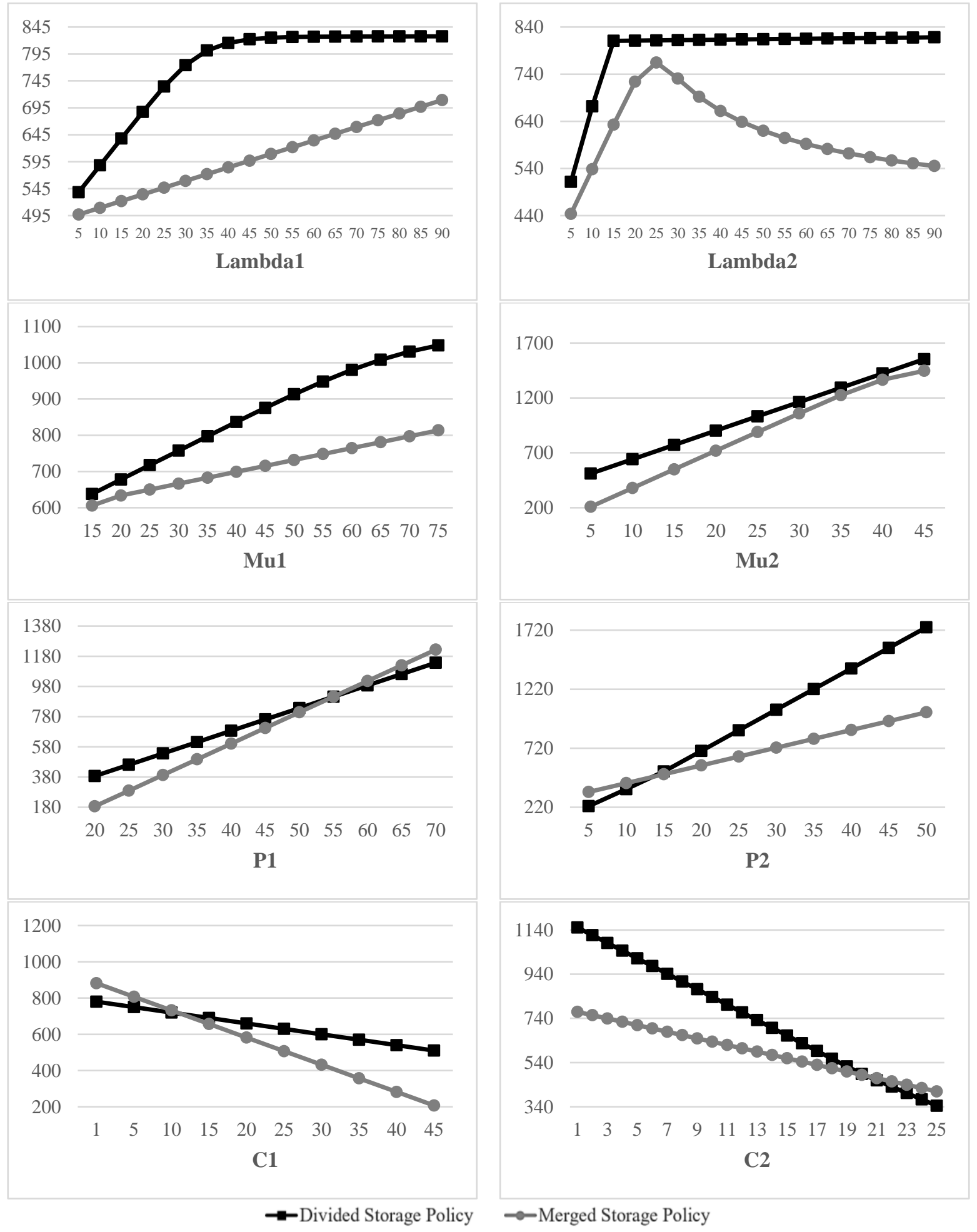

*The horizontal axis represents the changing parameter; all the other parameters are set at their center value based on table 7.

**In all cases, the vertical axis represents the total profit.

$* * *$ As $\lambda_{2}$ increases, the total profit for the merged storage strategy decreases as a result of congestion in the warehouse for having too many type 2 products.

Figure 4: Comparing the effect of changing the parameter values on the total profit of the two storage policies 


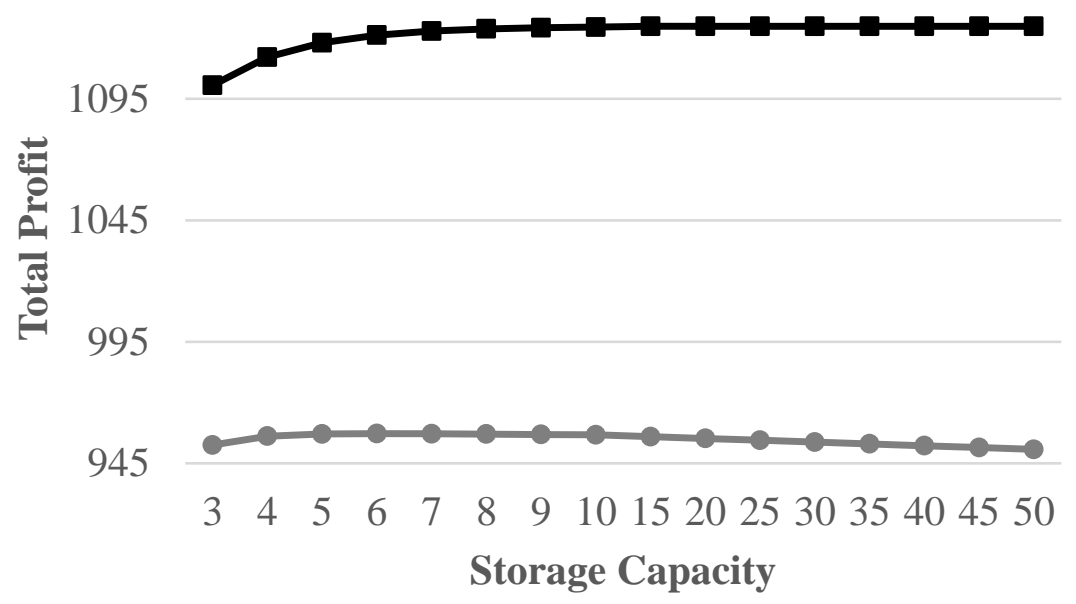

$\rightarrow-D i v i d e d$ Capacity Profit - -Merged Capacity Profit

Figure 5: Visualization of table 9

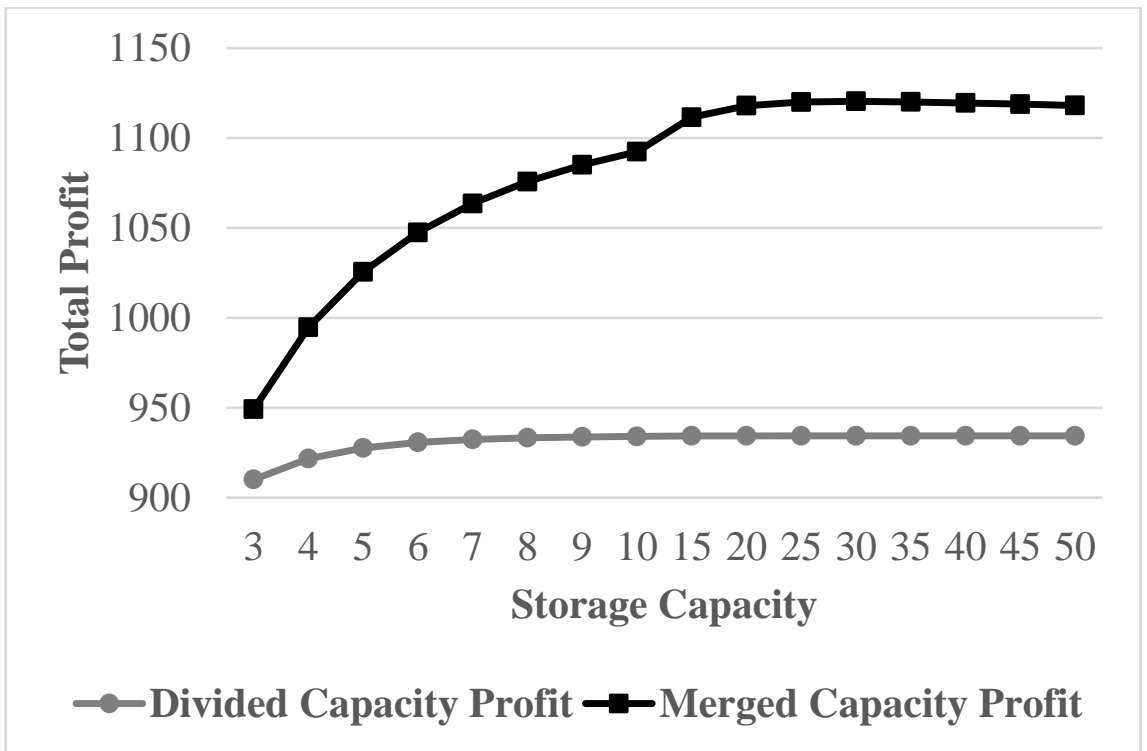

Figure 6: Visualization of table 10 